\title{
CONTENIDOS SOBRE LA «POLÍTICA DE EMPLEO» EN LA ASIGNATURA DE DERECHO DEL TRABAJO Y LA SEGURIDAD SOCIAL
}

\author{
Miguel Ángel Gómez SALAdo \\ Doctorando en Ciencias Jurídicas y Sociales. Departamento de Derecho del \\ Trabajo y de la Seguridad Social \\ magsalado@uma.es \\ Universidad de Málaga
}

Resumen: La estructura de los contenidos del tema 7 (titulado «Política de empleo y política de colocación») de la asignatura Derecho del Trabajo y la Seguridad Social que se imparte en el Grado en Trabajo Social de la Universidad de Málaga- desde el Departamento de Derecho del Trabajo y de la Seguridad Social ha tenido como propósito hacer de este tema un lugar para el análisis crítico del concepto de política de empleo, el marco jurídico-normativo de la política de empleo, las medidas existentes para el fomento del empleo, y la política de colocación.

En los diferentes epígrafes del presente artículo se presentan en líneas generales los contenidos y la teoría científica en que se apoya nuestro método de trabajo, haciendo especial hincapié en la necesidad de profundizar en los próximos cursos (esta profundización se presenta como propuesta de mejora) en los contenidos sobre la dimensión local de las políticas de empleo, reconociendo el considerable papel que desempeñan las entidades locales en torno al empleo debido a su cercanía a las necesidades de los ciudadanos.

Palabras clave: Aprendizaje, contenidos, dimensión local, políticas de empleo, y teoría científica de la política de empleo.

Abstract: The structure of the contents of theme 7 (entitled «Employment policy and placement policy») of the subject Labor Law and Social Security -taught in the Degree in Social Work of the University of Malaga- from the Department of Labor and Social Security Law has the purpose of making this topic a place for critical analysis of the employment policy concept, the legal-normative framework of employment policy, the existing measures for the promotion of employment, and the placement policy.

In the different sections of this article, the contents and the scientific theory on which our method of work is supported are presented in general terms, with special emphasis on the need to deepen in the next courses (this deepening is presented as a proposal for improvement) in the contents on the local dimension of employment policies, recognizing the considerable role played by local entities in employment due to their proximity to the needs of citizens.

REJIE Nueva época: Revista Jurídica de Investigación e Innovación Educativa Núm.17, Enero 2018, pp. 113-126

[En línea] http://www.revistas.uma.es/index.php/rejie 
Keywords: Learning, content, local dimension, employment policies, and scientific theory of employment policy.

Sumario: 1. Consideraciones iniciales. 2. La asignatura de Derecho del Trabajo y la Seguridad Social. 3. Los contenidos y la teoría científica del tema «Política de empleo». 4. La importancia de los contenidos sobre la dimensión local de las políticas de empleo: propuesta de mejora. 5. Conclusiones.

\section{Consideraciones iniciales}

El plan de estudios de las enseñanzas conducentes a la obtención del título universitario oficial de graduado o graduada en trabajo social ${ }^{1}$ por la Universidad de Málaga (vinculado a la rama de conocimiento de ciencias sociales y jurídicas) destaca la versatilidad de los estudios referidos y hace un especial hincapié en el estrecho vínculo de este título con la justicia social y la atención de las necesidades de las personas en sus diferentes ámbitos: social, familiar y laboral. En este sentido, la asignatura de Derecho del Trabajo y la Seguridad Social aporta al alumno una serie de conocimientos y competencias que, si bien no les convertirán en verdaderos expertos en normas laborales, les permitirán conocer los derechos y deberes de lo que se puede denominar «ciudadanía social» ${ }^{2}$.

Como se verá más adelante, uno de los temas que se imparten en esta asignatura viene referido a la política de empleo. No cabe duda de que un estudio intensivo de este tema puede ayudar a los graduados en trabajo social a estar en condiciones de asesorar y orientar mejor a todas aquellas personas que quieren y necesitan encontrar un trabajo y tienen mayores dificultades para encontrarlo.

En este trabajo se enfatiza la necesidad de profundizar en los contenidos sobre la políticas de empleo, pero más concretamente en los contenidos sobre la dimensión local de las políticas de empleo, reconociendo el considerable papel que desempeñan las entidades locales en torno al empleo debido a su cercanía a las necesidades de los ciudadanos.

\section{La asignatura de Derecho del Trabajo y la Seguridad Social}

Desde el punto de vista temático, la asignatura de Derecho del Trabajo y la Seguridad Social que se imparte en el Grado en Trabajo Social (UMA) tiene una gran amplitud. Conforme a lo dispuesto en la guía docente ${ }^{3}$ de la misma, esta asignatura

\footnotetext{
1 Puede consultarse el plan de estudios de las enseñanzas conducentes a la obtención del título universitario oficial de graduado o graduada en trabajo social por la Universidad de Málaga en el siguiente enlace: https://www.uma.es/grado-en-trabajo-social.

${ }^{2}$ Este término fue originariamente acuñado por Thomas Humphrey Marshall en su obra Ciudadanía y Clase social, publicada en el año 1950.

3 La Guía Docente de una asignatura es un documento público dirigido, fundamentalmente, a los estudiantes y escrito con un lenguaje muy claro, en el que se concreta, para un determinado curso
} 
pretende abordar, además del concepto del Derecho del Trabajo, las relaciones incluidas y excluidas de dicha noción, así como las relaciones laborales de carácter especial, la normativa que resulta de aplicación, y los criterios y principios de aplicación de la normativa laboral ${ }^{4}$.

Por otro lado, cabe destacar que en esta asignatura se presta también especial atención a los contratos de trabajo, la determinación de las condiciones de trabajo, las alteraciones y vicisitudes de la relación laboral, la extinción de la relación laboral y la seguridad social. Y a todo lo anterior hay que añadir la política de empleo -cuestión que analizaremos más adelante con un mayor detenimiento-.

Por ello, el programa completo de la asignatura se concreta en siete temas centrales (todos ellos de diferente entidad y amplitud) que, de una forma ordenada, se estructuran como sigue:

\begin{tabular}{|l|l|}
\begin{tabular}{|l|}
\multicolumn{2}{|c|}{ Contenidos de la asignatura Derecho del Trabajo y la Seguridad Social } \\
\hline
\end{tabular} \\
\hline Tema 1 & Programa completo \\
\hline Tema 2 & El contrato de trabajo \\
\hline Tema 3 & Determinación de las condiciones de trabajo \\
\hline Tema 4 & Alteraciones y vicisitudes de la relación laboral \\
\hline Tema 5 & La extinción de la relación laboral \\
\hline Tema 6 & La seguridad social \\
\hline Tema 7 & Política de empleo y política de colocación \\
\hline
\end{tabular}

El objetivo fundamental de esta asignatura es familiarizar a los futuros graduados en trabajo social con los derechos y con los deberes laborales más básicos de los trabajadores y de los empresarios. Para el cumplimiento de este objetivo, la asignatura tiene un enfoque eminentemente práctico. Con ello, se persigue «cierta destreza en la utilización de recursos y habilidades de búsqueda de información jurídica básica (legal, doctrinal o jurisprudencial) en materia de Derecho del Trabajo en bases de datos

académico, la planificación docente de una asignatura y toda la información necesaria para su seguimiento. Constituye el resultado del compromiso del equipo docente que la va a impartir y del Departamento al que se encuentra adscrita, avalado por la Universidad a través de los órganos con competencia en la aprobación de la programación docente. Aunque no se trate de un concepto recogido con esta denominación en la legislación vigente, el proceso de adaptación al Espacio Europeo de Educación Superior (EEES) ha propiciado su implantación y su uso entendiendo que la planificación de una asignatura en las enseñanzas universitarias implica atender a más aspectos de los que habitualmente se recogían en el denominado "programa" de la asignatura y, sobre todo, un cambio de enfoque en su elaboración, que ahora se plantea desde la perspectiva del estudiante. Por ello, la Guía Docente debe estar centrada en el desarrollo de las competencias propias de la asignatura y en el trabajo que el alumnado debe desarrollar para adquirir esas competencias.

${ }^{4}$ En el temario de la presente asignatura se hace especial hincapié en la regulación de la relación individual de trabajo. 
virtuales, además de cierta autonomía en la conversión individual de dicha información en conocimiento» ${ }^{5}$.

En la medida en que se trata de una asignatura de contenido jurídico, mantiene un estrecho vínculo con otras asignaturas de índole legal que están recogidas en el plan de estudios y que se cursan en el Grado en Trabajo Social, como el «Derecho de Familia», la «Protección jurídica del menor»o las «Bases de Derecho Administrativo», en tanto que las habilidades adquiridas en el manejo de normas, jurisprudencia o doctrina jurídica resultan provechosas para el estudio de las demás disciplinas jurídicas.

Mediante el cumplimiento del programa que arriba se detalla y la participación de los alumnos en las clases (teóricas y prácticas) y en el resto de actividades presenciales y no presenciales que se organizan en el aula docente, se procura que los alumnos adquieran las siguientes competencias que encajan completamente en los perfiles profesionales a los que se enfoca el Grado en Trabajo Social:

\begin{tabular}{|c|c|}
\hline \multicolumn{2}{|r|}{ Competencias } \\
\hline \multicolumn{2}{|r|}{ 1. Competencias generales y básicas } \\
\hline \multicolumn{2}{|r|}{ Descripción } \\
\hline 1.1. & $\begin{array}{l}\text { Que los estudiantes sepan aplicar sus conocimientos a su trabajo o } \\
\text { vocación de una forma profesional y posean las competencias que } \\
\text { suelen demostrarse por medio de la elaboración y defensa de } \\
\text { argumentos y la resolución de problemas dentro de su área de } \\
\text { estudio. }\end{array}$ \\
\hline 1.2 & $\begin{array}{l}\text { Que los estudiantes tengan capacidad de reunir e interpretar datos } \\
\text { relevantes para emitir juicios que incluyan una reflexión sobre temas } \\
\text { importantes de índole social, científico o ético. }\end{array}$ \\
\hline 1.3 . & $\begin{array}{l}\text { Que los estudiantes puedan transmitir información, ideas, problemas } \\
\text { y soluciones a un público tanto especializado como no. }\end{array}$ \\
\hline \multicolumn{2}{|r|}{ 2. Competencias específicas } \\
\hline & Descripción \\
\hline 2.1. & $\begin{array}{l}\text { Intervenir con personas, familias, grupos, organizaciones y } \\
\text { comunidades para ayudarles a tomar decisiones bien fundamentadas } \\
\text { acerca de sus necesidades, circunstancias, riesgos, opciones } \\
\text { preferentes y recursos. }\end{array}$ \\
\hline 2.2. & $\begin{array}{l}\text { Utilizar la mediación como estrategia de intervención destinada a la } \\
\text { resolución alternativa de conflictos. }\end{array}$ \\
\hline 2.3 . & $\begin{array}{l}\text { Defender a las personas, familias, grupos, organizaciones y } \\
\text { comunidades y actuar en su nombre si la situación lo requiere. }\end{array}$ \\
\hline 2.4. & Administrar y ser responsable de su propio trabajo asignando \\
\hline
\end{tabular}

\footnotetext{
${ }^{5}$ Tal y como se expone, de forma expresa, en el programa de la asignatura.
} 
prioridad.

\section{Los contenidos y la teoría científica del tema «Política de empleo»}

Centrándonos ahora en los contenidos del tema de la política de empleo que se imparte a los estudiantes del Grado en Trabajo Social, debemos comenzar refiriéndonos al art. 40 de la vigente Constitución Española, precepto en el cual se dispone que los poderes públicos de manera especial realizarán una política orientada al pleno empleo ${ }^{6}$. Este desarrollo de la política de empleo se recoge en nuestro derecho nacional en el Real Decreto Legislativo 2/2015, de 23 de octubre, por el que se aprueba el texto refundido de la Ley del Estatuto de los Trabajadores ${ }^{7}$, que conforma el núcleo normativo fundamental de la política de empleo en España. Ahora bien, también hay que considerar el resto de normas en materia laboral, tributaria y de Seguridad Social, que intervienen como instrumentos legales de apoyo.

Si se tiene en cuenta lo dispuesto en los arts. 40 y 41 de la Constitución Española, la política de empleo es el conjunto de decisiones adoptadas por el Estado y las comunidades autónomas que tienen por finalidad el desarrollo de programas y medidas tendentes a la consecución del pleno empleo, así como la calidad en el empleo, a la adecuación cuantitativa y cualitativa de la oferta y demanda de empleo, a la reducción y a la debida protección de las situaciones de desempleo (véase el art. $1^{8}$ del Real Decreto Legislativo 3/2015, de 23 de octubre, por el que se aprueba el texto refundido de la Ley de Empleo ${ }^{9}$ ).

En el marco de la política de empleo hay que tener que tener en cuenta a diversos actores: la Unión Europea, el Estado, las Comunidades Autónomas y las entidades locales, si bien nos centraremos en estas últimas.

\footnotetext{
${ }^{6}$ El art. 40 de la Constitución Española recoge expresamente lo siguiente: 1. Los poderes públicos promoverán las condiciones favorables para el progreso social y económico y para una distribución de la renta regional y personal más equitativa, en el marco de una política de estabilidad económica. De manera especial realizarán una política orientada al pleno empleo; 2. Asimismo, los poderes públicos fomentarán una política que garantice la formación y readaptación profesionales; velarán por la seguridad e higiene en el trabajo y garantizarán el descanso necesario, mediante la limitación de la jornada laboral, las vacaciones periódicas retribuidas y la promoción de centros adecuados.

${ }^{7}$ BOE núm. 255 de 24 de octubre de 2015.

${ }^{8}$ Que recoge literalmente lo siguiente: «Teniendo en cuenta lo establecido en los artículos 40 y 41 de la Constitución Española, la política de empleo es el conjunto de decisiones adoptadas por el Estado y las comunidades autónomas que tienen por finalidad el desarrollo de programas y medidas tendentes a la consecución del pleno empleo, así como la calidad en el empleo, a la adecuación cuantitativa y cualitativa de la oferta y demanda de empleo, a la reducción y a la debida protección de las situaciones de desempleo. La política de empleo se desarrollará, dentro de las orientaciones generales de la política económica, en el ámbito de la estrategia coordinada para el empleo regulada por el Tratado de Funcionamiento de la Unión Europea».

${ }^{9}$ BOE núm. 255 de 24 de octubre de 2015.
} 


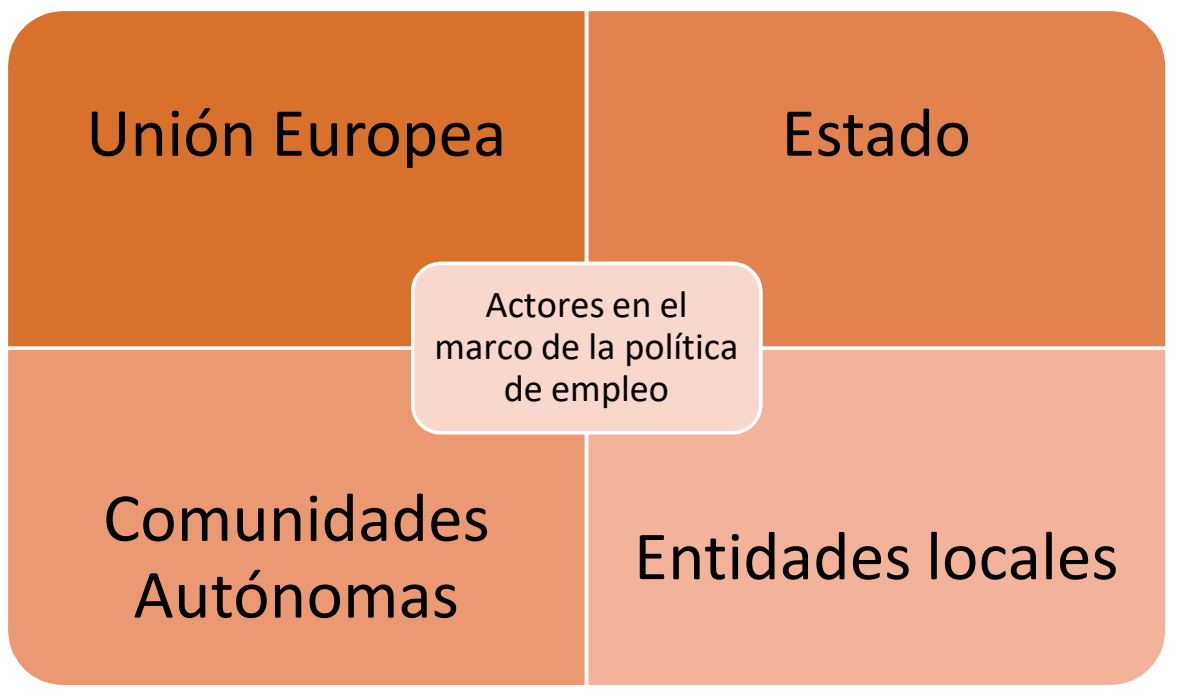

Como ya se ha dicho, la Unión Europea es uno de los actores esenciales, ya que tiene competencias especiales respecto a la coordinación de las políticas de empleo de los Estados miembros, en particular definiendo las orientaciones de dichas políticas ${ }^{10}$, tal y como se desprende del art. 5 del TFUE. Para definir las orientaciones de las políticas de empleo, la Unión Europea elabora las directrices (u orientaciones) de la política de empleo que deben aplicarse en los diferentes Estados miembros, las cuales se definen con un gran nivel de detalle y se renuevan cada año (art. 148 del Tratado de Funcionamiento de la Unión Europea). Asimismo, la Unión Europea desempeña un papel importante en lo que respecta a la financiación de los planes y acciones nacionales que se destinan al fomento del empleo y a la formación profesional, mediante los conocidos fondos estructurales.

Pero la competencia y la responsabilidad en materia de políticas de empleo corresponden fundamentalmente a dos actores principales: el Estado y las Comunidades Autónomas. Mientras que el primero de ellos, es decir, el Estado se encarga de la planificación y la ordenación, las Comunidades Autónomas que cuentan con competencias y con funciones de ejecución de la política estatal y de puesta en marcha de planes propios de empleo y formación.

Ahora bien, los entes locales en España también tienen un papel esencial, ya que, aunque la Constitución no les haya otorgado competencias específicas en materia de empleo, sí que pueden colaborar con el resto de niveles y participar en la ejecución y aplicación de programas y políticas activas de empleo, sin obviar que pueden poner en marcha acciones específicas siempre dentro de su propio ámbito de actuación.

Tras exponer un resumen de los contenidos del tema relativo a las políticas de empleo que se estudia en la asignatura de Derecho del Trabajo y la Seguridad Social y tras introducir a los principales actores (la Unión Europea, el Estado, las Comunidades Autónomas y las entidades locales) que intervienen en las políticas de empleo, vamos a presentar en el siguiente apartado la importancia del estudio por parte de los alumnos de la dimensión local de las políticas de empleo.

\footnotetext{
${ }^{10}$ Según se desprende del art. 5 del Tratado de Funcionamiento de la Unión Europea.
} 


\section{La importancia de los contenidos sobre la dimensión local de las políticas de empleo: propuesta de mejora}

Hasta el momento, hemos tratado de presentar los contenidos del tema referido a la política de empleo, pero en este nuevo apartado exponemos y desarrollaremos los contenidos y la teoría científica sobre la dimensión local de las políticas de empleo que, a nuestro parecer, son necesarios para ofrecer a los alumnos del Grado en Trabajo social en particular, y a los alumnos de las disciplinas sociales y jurídicas en general, una perspectiva de lo local, haciendo hincapié en temáticas relacionadas con el desarrollo local y el fomento del empleo en contextos territoriales y socioeconómicos diferenciados. Y para ello, reconoceremos el importante papel que desempeñan las entidades locales en torno al empleo debido a su cercanía a las necesidades de los ciudadanos.

Pues bien, a continuación tratamos de desarrollar los contenidos ${ }^{11}$ de la dimensión local de las políticas de empleo que acabamos de subrayar como necesarios para formar y cualificar al alumnado en temáticas relacionadas con el empleo local y el desarrollo de los diferentes territorios:

\subsection{Una visión general de las políticas de creación de empleo}

Según un reciente informe de la Organización Internacional del Trabajo (OIT), denominado «Perspectivas Sociales y del Empleo en el Mundo: Tendencias 2016» ${ }^{12}$, España presenta de cara a los próximos años una modesta tendencia hacia la recuperación del empleo, pero el pronóstico en general no es favorable pues, aunque está previsto que la tasa de paro descienda al $19,3 \%$ en 2017 , lo cierto es que nuestro país seguirá posicionándose entre las economías desarrolladas peor posicionadas debido a la creación de empleo vulnerable, las elevadas tasas de desempleo juvenil y la carencia de avances sociales que combatan la precariedad laboral.

En este sentido, no deja de ser paradójico que dos de las problemáticas más arduas y costosas del panorama económico y social actual, como resultado de la simultaneidad de múltiples factores (la robotización del mercado de trabajo ${ }^{13}$, la tecnificación creciente

\footnotetext{
${ }^{11}$ El desarrollo de estos contenidos en los siguientes apartados tiene su origen en el siguiente trabajo: GómEZ SALADO, M. A.: «Las políticas de creación de empleo en el marco local: Algunas ideas como conclusión», en AA.VV., El empleo en el ámbito local, Granada, Comares, 2017, págs. 361-369.

${ }^{12}$ El estudio «Perspectivas sociales y del empleo en el mundo: Tendencias 2016», publicado en enero de 2016, muestra una previsión de los niveles de desempleo tanto de las economías desarrolladas como de las emergentes a nivel mundial. Asimismo, propone un número significativo de recomendaciones para incentivar la creación de empleo en los próximos años.

${ }^{13}$ La robotización del mercado de trabajo se entiende como la proliferación en el entorno profesional de máquinas, tecnologías inteligentes, programas informáticos, robots y sistemas que automatizan trabajos que tradicionalmente han desempeñado las personas. Según Brynjolfsson, E. Y MCAFEE, A.: «Destrucción creativa: La economía de la aceleración tecnológica y de la destrucción de empleos», en $L a$ carrera contra la máquina, Antoni Bosch, 2013, págs. 41 a 69, este fenómeno, que hasta ahora solo había
} 
de los puestos de trabajo y la inestabilidad económica derivada de la crisis, entre otros muchos), sigan siendo, la estructuralización del desempleo y el limitado ritmo de creación de empleo. De ahí el deber de los poderes públicos de velar por la presencia de un mercado de trabajo que funcione correctamente a través de políticas de creación de empleo $^{14}$, entendidas como aquellas actuaciones que toman los poderes públicos con el objetivo de crear empleo estable y de calidad, en consonancia con el propósito de alcanzar el pleno empleo recogido en el artículo 40.1 de la $\mathrm{CE}^{15}$ o de, al menos, corregir los principales desequilibrios del mercado de trabajo, a la vez que se disminuyan las acentuadas desigualdades sociales.

Algunos de los instrumentos nacionales y europeos que reconocen ese objetivo son: la Estrategia Española de Activación para el Empleo 2017-2020 ${ }^{16}$ y la Estrategia Europea para el Crecimiento y el Empleo (Europa 2020) ${ }^{17}$.

Mediante la aprobación de la Estrategia Española de Activación para el Empleo se delimita un nuevo escenario para las políticas de empleo, tanto a nivel estatal como territorial. Hay que resaltar que esta Estrategia, desde el punto de vista del desarrollo local como política de creación de empleo, sitúa el desarrollo económico territorial dentro de su marco de actuación y reconoce la importancia de las estrategias locales a efectos del aprovechamiento de nuevas oportunidades ligadas al aumento de la exportación y a las innovaciones tecnológicas, con lo cual resulta indispensable promover y gestionar en el marco local actuaciones encaminadas a la creación de empleo y la iniciativa empresarial.

Por otra parte, la política de creación de empleo desarrollada en España no puede ser ajena a la política y a las directrices de la Unión Europea. Desde este planteamiento, cabe destacar la Estrategia Europea para el Crecimiento y el Empleo, Europa 2020, cuya finalidad principal es transformar el modelo de crecimiento de la Unión Europea, de manera que se propicien las condiciones favorables para un tipo de crecimiento distinto, más inteligente, sostenible e integrador, basado en una economía con un elevado nivel

afectado las tareas más manuales, ha comenzado a colarse en un sinfín industrias más complejas generará más desigualdad, desempleo y una ruptura del orden social tradicional.

${ }^{14}$ Sea como fuere, es evidente que existe una cierta indefinición de la política de empleo en España, pues tan solo el artículo $1^{\circ}$ del Real Decreto Legislativo 3/2015, de 23 de octubre, por el que se aprueba el texto refundido de la Ley de Empleo (BOE núm. 255, de 24 de octubre de 2015) hace un intento de definirla, teniendo en cuenta lo dispuesto en los artículos 40 y 41 de la Constitución Española, como aquella política que tiene por finalidad "el desarrollo de programas y medidas tendentes a la consecución del pleno empleo, así como la calidad en el empleo, a la adecuación cuantitativa y cualitativa de la oferta y demanda de empleo, a la reducción y a la debida protección de las situaciones de desempleo". En este sentido, RODRÍGUEZ-PIÑERO ROYO, M.: «Política económica y política de empleo», en VV.AA., La política y el Derecho del Empleo en la nueva sociedad del trabajo, Consejo Andaluz de Relaciones Laborales, Sevilla, 2016.

${ }^{15}$ Los poderes públicos no solo deben desarrollar políticas de creación de empleo, sino que deben orientarlas al pleno empleo, el cual encuentra su justificación en el artículo 40.1 de la CE.

16 Se encuentra actualmente en fase de tramitación y pretende renovar la Estrategia Española de Activación para el Empleo 2014-2016, que fue aprobada por el Real Decreto 751/2014, de 5 de septiembre (BOE núm. 231, de 23 de septiembre de 2014) y que ya ha concluido.

${ }^{17}$ La Estrategia Europa 2020 se aplica a través de seis grandes orientaciones de política económica de los Estados miembros y la Unión (Recomendación del Consejo de 13 de julio de 2010) y de cuatro orientaciones de política de empleo de los Estados miembros (Decisión del Consejo de 21 de octubre de 2010), que sustituyen a las de la Estrategia de Lisboa. 
de empleo, es decir, una economía en la cual se creen más y mejores puestos de trabajo. Para alcanzar tal crecimiento, cabe dar cuenta de una forma de proceder en la que se consiga cumplir un objetivo primordial: aumentar hasta el 75\% la tasa de empleo de la población de entre 20 y 64 años, mediante una mayor participación de los jóvenes, los trabajadores de mayor edad y los trabajadores de menor cualificación.

Ahora bien, realmente, estas políticas de empleo nacionales y europeas no están logrando el resultado previsto de estimular un entorno que posibilite la creación de más y mejores puestos de trabajo. A nuestro parecer, resulta evidente la ineficacia a la hora de responder a un problema concreto, el de la generación de empleo de calidad -ya sea de forma directa o indirecta-, pues han proliferado los empleos temporales y a tiempo parcial, escasamente remunerados y caracterizados por una notable precariedad en las condiciones laborales de los trabajadores, es decir, aspectos que, al mismo tiempo, reducen considerablemente la estabilidad laboral necesaria para una recuperación real.

A la vista del fracaso de las anteriores políticas mencionadas, la solución podría encontrarse en la apuesta por políticas de creación de empleo en el marco local debido a la cercanía de los agentes locales a las necesidades de los ciudadanos, de manera que se transforme el análisis en medidas concretas y viables a corto, medio y largo plazo que reviertan en la generación de empleo local de calidad.

\subsection{La dimensión local en las políticas de creación de empleo}

Con lo anterior es muy importante insistir en dos líneas fundamentales y claramente interrelacionadas que se desprenden del tratamiento de la generación de empleo a escala local.

La primera, la de aclarar si el ámbito local es adecuado para llevar a cabo las políticas de empleo. En segundo y último lugar, e íntimamente relacionado con lo anterior, es importante señalar una serie de propuestas que logren activar la creación de empleo.

Veamos, si quiera brevemente, y a efectos de clarificar un poco estas cuestiones, los siguientes apartados:

\subsubsection{Un enfoque integrado de las políticas de creación de empleo}

Por lo que concierne, con algún detalle más, a la creación de empleo, que desde hace algún tiempo se ha convertido en uno de los objetivos más perseguidos por la sociedad, conviene responder al siguiente interrogante: ¿es el ámbito local el medio más adecuado para llevar a cabo las políticas de creación de empleo?

Centrando la atención, por tanto, en la Administración Pública Local, y especialmente en la estimulación de la economía local y en búsqueda de un mayor bienestar social, cabría poner de manifiesto que sus actuaciones en un gran número de ocasiones convergen con las actuaciones y competencias de otras administraciones de 
mayor ámbito económico y territorial. Y esto es así porque, en el marco de la Unión Europea, las políticas de empleo han sido tradicionalmente competencia de los Estados, de forma que el papel de las entidades locales en materia de políticas de empleo se ha visto reducido a la mera colaboración con el Estado y las Comunidades Autónomas en la aplicación de medidas convenidas por $\operatorname{estos}^{18}$.

Consecuentemente, las entidades locales tienen un margen de actuación limitado, si bien pueden adoptar algunas medidas eficaces en materia de políticas de creación de empleo destinadas a aumentar la cantidad de empleos disponibles a nivel local con diversos instrumentos políticos, como la cesión gratuita de locales públicos durante un intervalo de tiempo, el fomento de la exportación de la agricultura ecológica $\mathrm{o}$ determinados incentivos para que los empleadores realicen mayores inversiones en nuevas tecnologías. No obstante, también es necesario centrarse en la calidad de los empleos locales, creando empleos reales y sostenibles en el tiempo. Todo ello en virtud de la autonomía local a la que se refiere el artículo 137 de la Constitución Española.

Sea como fuere, mediante la realización de políticas a escala local pueden emprenderse acciones efectivas y mecanismos en beneficio de la sociedad que atiendan a los distintos tipos de necesidades y respondan a los problemas y oportunidades de carácter económico y social, fundamentalmente, en relación a la generación de empleo, siendo múltiples los recursos de los que disponen las entidades locales para materializar estas políticas: los recursos humanos -activos más valiosos de cualquier organización-, políticos, financieros, sociales, culturales, institucionales, las infraestructuras, etc.

En cualquier caso, la territorialización de las políticas de empleo posibilita la descentralización de las mismas y una mejor atención a las particularidades de los diversos territorios. En esta línea, debemos admitir que las políticas deben realizarse desde la proximidad, reconociendo el considerable papel que desempeñan las entidades locales a la hora de crear empleo debido a su cercanía a las necesidades de los ciudadanos, y haciendo que los ciudadanos sean partícipes a los efectos de lograr una mayor efectividad.

Cierto es que las políticas de creación de empleo deben realizarse desde la proximidad al ciudadano, pero al mismo tiempo deben realizarse de forma coordinada con los distintos niveles administrativos, sobre la base de un diagnóstico compartido de los problemas y de los aspectos positivos de los que se parte. En este sentido, el Real Decreto Legislativo 3/2015, de 23 de octubre, por el que se aprueba el texto refundido de la Ley de Empleo se refiere a la necesaria colaboración de las distintas administraciones públicas y resalta que las políticas de empleo, en su diseño y modelo de gestión, deben tener en cuenta su dimensión local para ajustarlas a las necesidades del territorio, de forma que favorezcan y apoyen las iniciativas de generación de empleo de calidad en el ámbito local.

\footnotetext{
${ }^{18}$ En particular, es preciso mencionar que las posibilidades de actuación de las entidades locales en lo relativo al empleo dependen de lo dispuesto en los artículos 137 y 140 de la Constitución Española, en los distintos Estatutos de Autonomía y en las normas estatales, particularmente, la Ley 7/1985, de 2 de abril, Reguladora de las Bases de Régimen Local y el RD Legislativo 781/1986, de 18 de abril por el que se aprueba el Texto Refundido de las disposiciones legales vigentes en materia de Régimen Local.
} 
Y mención especial merece el artículo 4 de dicha norma relativo a la colaboración con las entidades locales. En su redacción, aclara que la norma comprende al Municipio, a la Provincia, a las Islas, a las Comarcas u otras entidades que agrupen varios Municipios, a las Áreas Metropolitanas y a las Mancomunidades de Municipios, es decir, a todos los entes locales detallados en el artículo 3 de la Ley 7/1985, de 2 de abril, reguladora de las Bases del Régimen Local ${ }^{19}$.

Al hilo de estos últimos planteamientos y a mi parecer, si se pierde la perspectiva de un enfoque integrado para poder establecer mecanismos y articular estrategias que incidan en los sectores económicos locales y, fundamentalmente, en la creación de empleo, sin coordinación ni colaboración con el resto de actores (entre otros, la Unión Europea, el Estado y las Comunidades Autónomas), y la creación de empleo se plantea como una política aislada, monopolio de las entidades locales, todo habrá fracasado.

Centrándonos en este asunto, quizás deba irse un poco más allá, es decir, no considerar únicamente un enfoque integrado de la política de creación de empleo con todos los actores, sino, además, adoptar un planteamiento integrado de todas y cada una de las políticas que se desarrollan a escala local. No habría que convertir, así, la política de empleo en la piedra angular de las políticas públicas y situar la creación de empleo como el principal objetivo a cumplir, sino que habría que conseguir que todas las políticas se encuentren enfocadas y concebidas para la creación de empleo. Parece este un planteamiento muy coherente para reducir los elevados índices de desempleo de carácter estructural $^{20}$-es decir, aquel que permanece al margen del ciclo económicoque presentan la mayor parte de los territorios que conforman nuestro país.

En todo caso, aflora aquí la dificultad para lograr el éxito de estas políticas con las particularidades de las distintas localidades y territorios (diferentes contextos económicos, políticos y sociales) ${ }^{21}$. La dificultad aparece cuando no se considera este enfoque integrado, de ahí la necesidad de que en el diseño de las políticas de creación de empleo se sea especialmente cuidadoso con este encuadre.

Por todo lo expuesto anteriormente, podemos responder al interrogante que se planteó en un primer momento, concluyendo que el ámbito local es idóneo para llevar a cabo políticas destinadas a la generación de empleo debido a su cercanía a las necesidades de los ciudadanos. No obstante, como se ha indicado, las políticas deben lograr una mayor integralidad y coherencia, así como no desvincularse de las actuaciones que se realizan en otros niveles.

\footnotetext{
${ }^{19}$ Literalmente señala que: 1) «son entidades locales territoriales: a) el Municipio, b) la Provincia y c) la isla en los archipiélagos balear y canario»; y 2) asimismo, dispone que gozan «de la condición de Entidades Locales: a) las Comarcas u otras entidades que agrupen varios Municipios, instituidas por las Comunidades Autónomas de conformidad con esta Ley y los correspondientes Estatutos de Autonomía, $b$ ) las Áreas Metropolitanas y c) las Mancomunidades de Municipios».

${ }^{20}$ Tal y como refiere MONTILla PÉREZ, S.: «Diferencias individuales de los desempleados y constructos motivacionales: una relación de interdependencia predictora del desempleo estructural», Revista de psicología del trabajo y de las organizaciones, vol. 21, núm. 3, 2005, págs. 269-298.

${ }^{21}$ En muchos casos, el hecho de que una política destinada a la generación de empleo funcione bien en un determinado territorio o con un grupo específico (jóvenes, mujeres, personas con discapacidad, desempleados de larga duración, beneficiarios de prestaciones por desempleo, entre otros) no significa que deba funcionar igual de bien en otros.
} 
De igual modo, debe, no obstante priorizarse el objetivo del empleo frente a cualquier otro que pueda entenderse como secundario. De esta manera, finalidades como la sostenibilidad presupuestaria o la consolidación de las cuentas públicas se tendrían que entender como una vía para garantizar la viabilidad futura de las propias administraciones, pero este objetivo, en sí mismo, no puede conseguirse si a largo plazo no se consolida el empleo y, para ello, instrumentos como los previstos para evitar la duplicidad o garantizar la sostenibilidad deben ser siempre relativizados en función de la potencialidad o del marco o destinatarios que aquellas medidas específicas presenten.

\subsubsection{Propuestas para activar la creación de empleo desde el ámbito local: un enfoque práctico de su contribución al desarrollo local}

Es verdad que la crisis ha causado estragos en todos los sectores de la economía, lo cual precisamente se ha intensificado en el sector servicios, en el comercio y en el sector del turismo, teniendo en cuenta que cualquier crisis económica transforma los hábitos de los consumidores. Desafortunadamente, no es nada sencillo encontrar sectores de la economía que no hayan sufrido la pérdida de puestos de empleo.

Por ello es conveniente programar políticas que promuevan el desarrollo territorial a escala local y que propongan medidas específicas para crear nuevos puestos de trabajo en el término local, por ejemplo, mediante la explotación de nuevos yacimientos de empleo $^{22}$.

Hace falta, pues, que el ámbito local identifique aquellas áreas de la economía que, indiscutiblemente, practicarán un crecimiento en los próximos años, y para las cuales las comunidades locales deberían disponer su mano de obra.

En primer lugar, una de estas áreas claves a efectos de la generación de empleo es el sector en auge de la asistencia médica y social, debido al envejecimiento de la población. Concretamente, hay una serie de actividades cuyos protagonistas son las personas dependientes -mayores o con alguna discapacidad-, como son la atención a este colectivo, la limpieza y el cuidado del hogar y de la alimentación, la asistencia geriátrica y residencial, las reparaciones del hogar y la compra diaria, entre otras muchas actividades, que comúnmente ha desarrollado algún miembro de la familia tradicionalmente de sexo femenino-, y que están actualmente siendo transferidas a actores y agentes externos por los cambios en el papel que desempeña la mujer en el ámbito familiar y laboral y por la transformación de la familia tradicional.

Otro sector que puede generar empleo a nivel local es el de las actividades turísticas en el medio local, especialmente rural, con las cuales se puede atraer a nuevos emprendedores. Sin duda, las excelencias gastronómicas de las distintas zonas

\footnotetext{
${ }^{22}$ Los nuevos yacimientos de empleo, también conocidos como filones de ocupación, son el término utilizado por el Libro Blanco de Delors para describir aquellas actividades laborales que, por un lado, ayudan a combatir el desempleo y que, por otro lado, contribuyen a satisfacen las necesidades sociales parcialmente atendidas o sin atender. En este sentido, VillalongA, A.: «Los nuevos yacimientos de empleo: una oportunidad para crear empleo y satisfacer nuevas necesidades sociales», Revista electrónica de geografía y ciencias sociales, núm. extra 6, 2002.
} 
geográficas propician el crecimiento de un turismo que reivindica una especialización en cocina, gastronomía y dietética.

Debe tenerse también en cuenta que ello posibilitaría un crecimiento ligado al aumento de la exportación y a las innovaciones tecnológicas e iniciativas empresariales.

Y, en último lugar, y no por ello menos importante, el creciente interés social por el medio ambiente y por el enorme valor ecológico. De un lado, el interés medioambiental implica la necesidad de optimizar la gestión pública y privada de los residuos, del agua, del aire, así como invertir en la reducción de la contaminación. De otro lado, el creciente interés por el valor ecológico y los nuevos modelos de consumo exigen una inversión en la agricultura -especialmente en la agricultura ecológica- y en las innovaciones de la industria alimentaria. A nivel local, la explotación de estas actividades medioambientales y ecológicas puede generar puestos de empleo y crecimiento a corto, medio y largo plazo.

Por todo lo expuesto con anterioridad, existen numerosas áreas claves para contribuir a la generación de empleo desde el ámbito local. Como se verá a continuación existen numerosas medidas e iniciativas emprendedoras aprovechables en el ámbito local para generar empleo y de gestión en el ámbito local:

En primer lugar, cabe destacar la creación de un plan local de empleo, que garantice la inserción laboral de aquellas personas que quieran acceder a un empleo ${ }^{23}$. Así pues, este plan se acompañaría de otras políticas de generación de empleo, con la finalidad de impulsar la creación de puestos de trabajo sostenibles y duraderos en sectores emergentes, así como puestos que no sean rentables para la iniciativa privada como la rehabilitación de espacios medioambientales y urbanísticos, además de su mantenimiento. En el marco de estos planes de empleo se desarrollaría un programa de formación con el objeto de mejorar la empleabilidad ${ }^{24}$.

En segundo lugar, medidas encauzadas al fomento del autoempleo, estimulando aquellas actividades laborales que se encuentren impulsadas por ideas innovadoras, así como el impulso de la economía social y de aquellos sectores generadores de empleo con posibilidades de carácter medioambiental, ecológico, cultural, artístico y gastronómico.

En este sentido, se deberán construir marcas fuertes y desarrollar ideas innovadoras, mediante las ventajas comparativas que presenta un determinado territorio para su promoción y comercialización en mercados nacionales e internacionales.

En tercer lugar, el desarrollo de planes de rehabilitación de espacios degradados del centro histórico y reconversión de locales públicos y espacios abandonados en

\footnotetext{
${ }^{23}$ Específicamente desempleados de larga duración y sectores con dificultades de acceso al empleo jóvenes, mujeres, mayores de 55 años y discapacitados-.

${ }^{24} \mathrm{Si}$ bien es cierto que incrementar los niveles de educación y formación, así como mejorar los conocimientos y competencias de los trabajadores, es una garantía para el empleo y para el empleo de calidad, también es cierto que el problema de la falta de empleos no se soluciona aunque las personas sean muy «empleables».
} 
residencias de atención para las personas mayores, centros de trabajo para personas con discapacidad, escuelas infantiles y escuelas locales de empresas ${ }^{25}$.

En esta línea, también se encuadra la cesión de suelo rústico para el desarrollo de iniciativas empresariales con retorno social, así como la cesión gratuita de locales públicos durante un período de tiempo con la finalidad de impulsar nuevas ideas de negocio. Véase el caso de las escuelas para el fomento de la agricultura, las granjas escuela y los centros de rehabilitación animal, entre otros.

En cuarto lugar, la habilitación de una serie de plataformas y portales on-line destinados al comercio tradicional del territorio, en cuyo caso es imprescindible contar con una adecuada estrategia de comunicación para la promoción y el estímulo del consumo.

En quinto lugar, el apoyo a la financiación a través de microcréditos o acciones que fomenten el acceso al crédito a través de acuerdos de colaboración con las entidades bancarias, o, en su defecto, la creación de cooperativas sociales públicas que den acceso al crédito.

No obstante, al margen de las medidas expuestas, existen otras medidas que pueden desarrollarse a nivel local para generar puestos de empleo, como pueden ser las bonificaciones a las empresas, las convocatorias de premios locales a las ideas o iniciativas empresariales más innovadoras, las medidas de atracción a la inversión extranjera con retorno social, etcétera.

En definitiva, a diferencia de lo que a veces se puede llegar a pensar, existe un amplio catálogo de opciones aplicables al ámbito local en el que las administraciones locales tienen mucho que aportar. Así, con lo expuesto en los apartados anteriores, podemos afirmar que las políticas de creación de empleo local son muy importantes para combatir las elevadas tasas de desempleo. Ahora bien, requieren de una gran implicación política y social.

\section{Conclusiones}

El análisis de los contenidos sobre la «política de empleo» en la asignatura de Derecho del Trabajo y la Seguridad Social -que se imparte en el Grado en Trabajo Social de la Universidad de Málaga- que se ha desarrollado en las páginas precedentes, nos ha permitido concluir que el acercamiento de la comunidad educativa a la teoría científica que trata de explicar la dimensión local de la política de empleo puede ser muy positivo, en la medida en que a través de este acercamiento se puede ofrecer a los alumnos del Grado en Trabajo social en particular, y a los alumnos de las disciplinas sociales y jurídicas en general, una perspectiva de lo local, haciendo especial hincapié en las temáticas relacionadas con el desarrollo local y el fomento del empleo en contextos territoriales y socioeconómicos diferenciados.

\footnotetext{
${ }^{25}$ A este respecto, resulta preciso destacar que estas escuelas locales de empresas, como la Escuela Municipal de Empresas de Málaga, proporcionan un apoyo clave para aquellas personas con inquietudes hacia diversos campos relacionados con el mundo de la empresa, sea cual sea su dimensión, ofreciendo determinados cursos, seminarios, consultorías y talleres adaptados a las últimas tendencias.
} 